\title{
A NOTE ON WEIGHTED SOBOLEV SPACES, AND REGULARITY OF COMMUTATORS AND LAYER POTENTIALS ASSOCIATED TO THE HEAT EQUATION
}

\author{
STEVE HOFMANN
}

(Communicated by J. Marshall Ash)

\begin{abstract}
We give a simplified proof of recent regularity results of Lewis and Murray, namely, that certain commutators, and the boundary single layer potential for the heat equation in domains in $\mathbb{R}^{2}$ with time dependent boundary, map $L^{p}$ into an appropriate homogeneous Sobolev space. The simplification is achieved by treating directly only the case $p=2$, but in a weighted setting.
\end{abstract}

\section{INTRODUCTION AND STATEMENT OF RESULTS}

Regularity results for certain commutators and layer potentials associated to the heat equation in domains in $\mathbb{R}^{2}$ with time dependent boundary have recently been obtained by Lewis and Murray [LM]. They showed that these operators are bounded from $L^{p}$ into an appropriate Sobolev space $I_{\alpha}\left(L^{p}\right)$. Their proof proceeds in two steps: First treat the case $p=2$, and then use a variety of real variable techniques to extend to the case $p \neq 2$. Their results are equivalent to the $L^{p}$ boundedness of certain "nonstandard" singular integrals that, in particular, need not map constants into BMO; thus the T1 Theorem does not apply, nor can one interpolate with an end point estimate to obtain the case $2<p<\infty$. Not surprisingly then, the second part of their program entails a not inconsiderable expenditure of effort, and it would therefore seem desirable to dispense with this step entirely. Fortunately, there is a way to do this: in the (possibly appocryphal) words of Rubio de Francia, " $L^{p}$ does not exist, only (weighted) $L^{2}$." In this note we will prove a weighted version of the $L^{2}$ result of [LM], from which most of the $L^{p}$ theory follows automatically (in particular, we obtain the case of principal interest in parabolic theory, namely, $\alpha=\frac{1}{2}$ for all $\left.p, 1<p<\infty\right)$. While the weighted results are new and perhaps of independent interest, our primary motivation in establishing them is to simplify the arguments of [LM].

Before stating our theorems, we need to recall some elementary facts about Littlewood-Paley theory in $\mathbb{R}^{n}$. Let $\psi \in C_{0}^{\infty}\left(\mathbb{R}^{n}\right)$ be radial, be supported in the unit ball, and have mean value zero. We define $Q_{s} f \equiv \psi_{s} * f$, where $\psi_{s}(x) \equiv$

Received by the editors May 1, 1991 and, in revised form, November 27, 1991.

1991 Mathematics Subject Classification. Primary 42A50, 35K05, 35B65; Secondary 42B25, $46 \mathrm{E} 35$. 
$s^{-n} \psi(x / s)$, and where $\psi$ has been normalized so that $\int_{0}^{\infty}(\hat{\psi}(s \xi))^{2} d s / s=1$ for all $\xi \in \mathbb{R}^{n}$ (this can be done since $\psi$ is radial). Thus $Q_{s}$ satisfies the "Calderon reproducing formula"

$$
\int_{0}^{\infty} Q_{s}^{2} \frac{d s}{s}=I .
$$

For $0<\alpha<1$, we define $\widetilde{Q}_{s} \equiv s^{-\alpha} I_{\alpha} Q_{s}$, where as usual $I_{\alpha}$ denotes the fractional integral operator

$$
\left(I_{\alpha} f\right)^{\wedge}(\xi) \equiv|\xi|^{-\alpha} \hat{f}(\xi) .
$$

Then, at least for test functions,

$$
\int_{0}^{\infty} \widetilde{Q}_{s}^{2} \frac{d s}{s}=C_{\alpha} I
$$

since

$$
0<\int_{0}^{\infty} s^{-2 \alpha}(\hat{\psi}(s))^{2} \frac{d s}{s}=C_{\alpha}<\infty, \quad 0<\alpha<1 .
$$

The latter inequality follows from the smoothness of $\hat{\psi}$ and the fact that $\hat{\psi}(0)=$ 0 (so, in particular, $|\hat{\psi}(s) / s|$ is bounded near the origin). If we set

$$
\hat{\tilde{\psi}}(|\xi|) \equiv|\xi|^{-\alpha} \hat{\psi}(|\xi|)
$$

then a routine computation shows that

$$
\left|\tilde{\psi}_{s}(x)\right| \leq \frac{C_{n, \alpha} s^{1-\alpha}}{(s+|x|)^{n+1-\alpha}} \quad \text { and } \quad\left|\nabla \tilde{\psi}_{s}(x)\right| \leq \frac{C_{n, \alpha} s^{1-\alpha}}{(s+|x|)^{n+2-\alpha}} .
$$

Thus,

$$
\left(\int_{0}^{\infty}\left|\tilde{\psi}_{s}(x)\right|^{2} \frac{d s}{s}\right)^{1 / 2} \leq C|x|^{-n}
$$

and

$$
\left(\int_{0}^{\infty}\left|\nabla \tilde{\psi}_{s}(x)\right|^{2} \frac{d s}{s}\right)^{1 / 2} \leq C|x|^{-n-1} .
$$

By vector-valued Calderon-Zygmund theory (see, e.g., [GR, Chapter V]), we then have, for all $w \in A_{2}$,

$$
\|\tilde{g}(f)\|_{2, w} \approx\|f\|_{2, w},
$$

where

$$
\tilde{g}(f) \equiv\left(\int_{0}^{\infty}\left|\widetilde{Q}_{s} f\right|^{2} \frac{d s}{s}\right)^{1 / 2} .
$$

Furthermore, the homogeneous weighted Sobolev space $I_{\alpha}\left(L_{u l}^{2}\right), w \in A_{2}$, can be given the norm

$$
\|f\|_{I_{\alpha}\left(L_{w}^{2}\right)} \equiv\left(\int_{\mathbb{R}^{n}} \int_{0}^{\infty}\left|Q_{s} f(x)\right|^{2} \frac{d s}{s^{1+2 x}} w(x) d x\right)^{1 / 2},
$$

because by (1.1) this last expression is comparable to $\|\tilde{f}\|_{2, w}$, where $f=$ $I_{\alpha}(\tilde{f})$. 
For $0<\alpha<1$, let (real-valued) $A \in I_{\alpha}(\mathrm{BMO})$, and consider the onedimensional operator

$$
K_{\alpha} f(x) \equiv \int_{\mathbb{R}} \frac{[A(x)-A(y)]^{2}}{|x-y|^{1+\alpha}} f(y) d y .
$$

As in [LM], our results for this operator can easily be extended to the boundary single layer potential for the heat equation in domains $\left\{\left(x_{1}, x_{2}\right): x_{1}>A\left(x_{2}\right)\right\}$, with $A=I_{1 / 2} a, a \in \mathrm{BMO}$. We shall return to this point in $\S 4$. Our principal result is the following.

Theorem 1.4. Let $A=I_{\alpha} a$, and suppose $w \in A_{1}$ if $0<a<1$ or $w \in A_{2}$ if $\frac{1}{2} \leq \alpha<1$. Then

$$
\left\|K_{\alpha} f\right\|_{I_{\alpha}\left(L_{w}^{2}\right)} \leq C_{\alpha}\|a\|_{*}^{2}\|f\|_{2, w} .
$$

As an almost immediate corollary, we recover, except for the case $1<p<2$, $0<\alpha<\frac{1}{2}$, the result of [LM, Theorem 3].

Theorem 1.5. Suppose $1<p<\infty$ if $\frac{1}{2} \leq \alpha<1$, or $2 \leq p<\infty$ if $0<\alpha<1$. Then

$$
\left\|K_{\alpha} f\right\|_{I_{\alpha}\left(L^{p}\right)} \leq C_{\alpha, p}\|a\|_{*}^{2}\|f\|_{p} .
$$

Proof of Theorem 1.5 (Modulo Theorem 1.4). If $\frac{1}{2} \leq \alpha<1$, then by Theorem 1.4 we have that $D^{\alpha} K_{\alpha}$ is bounded on $L_{w}^{2}, w \in A_{2}$, and therefore on $L_{w}^{p}$, $1<p<\infty, w \in A_{p}$, by Rubio de Francia's extrapolation theorem (see, e.g., [GR, Chapter IV]), where

$$
\left(D^{\alpha} f\right)^{-}(\xi) \equiv|\xi|^{\alpha} \hat{f}(\xi)
$$

If $0<\alpha<1$ then $D^{\alpha} K_{\alpha}$ is bounded on $L_{w}^{2}, w \in A_{1}$. In particular, by a result of Coifman and Rochberg [CR], we have for $u \in L^{(p / 2)^{\prime}}, p>2$, the inequality

$$
\int\left|D^{\alpha} K_{\alpha} f(x)\right|^{2} u(x) d x \leq C_{\alpha, \varepsilon}\|a\|_{*}^{4} \int|f(x)|^{2}\left(M\left(|u|^{1+\varepsilon}\right)\right)^{1 / 1+\varepsilon}(x) d x,
$$

for any positive $\varepsilon$. By choosing $1+\varepsilon<(p / 2)^{\prime}$, the $L^{p}$ boundedness, $p>2$, of $D^{\alpha} K_{\alpha}$ may be deduced by a standard duality argument. Theorem 1.5 follows.

We remark that the proof to follow will actually show that in the case $0<$ $\alpha<\frac{1}{2}$, one may take $w \in A_{1+2 \alpha}$ in Theorem 1.4 , and therefore by a slightly more involved duality argument, one obtains Theorem 1.5 for $p>2 /(1+2 \alpha)$. This is the best result that can be directly obtained by our method, which relies on the auxilary use of the Littlewood-Paley $g_{\lambda}^{*}$ function, with $\lambda<1+2 \alpha$. Since the full range of $p$ has already been treated in [LM], we shall content ourselves with Theorems 1.4 and 1.5 as stated.

In the next section, we give a transparent extension to the weighted setting of a result of Strichartz relating Carleson measures and $I_{\alpha}(\mathrm{BMO})$. In $\S 3$ we prove Theorem 1.4, and then in $\S 4$ we describe how this result may be extended to the boundary single layer potential. 


\section{2. $I_{a}(\mathrm{BMO})$ AND Weighted CARLESON MEASURES}

We first need a preliminary fact.

Lemma 2.1. With $0<\alpha<1$, the square function $g_{\alpha} f$ defined on $\mathbb{R}^{n}$ by

$$
g_{\alpha} f(x) \equiv\left(\int_{\mathbb{R}^{n}}\left|I_{\alpha} f(x+h)-I_{\alpha} f(x)\right|^{2} \frac{d h}{|h|^{n+2 \alpha}}\right)^{1 / 2}
$$

is bounded on $L_{w}^{2}, w \in A_{p(\alpha)}$, where $p(\alpha) \equiv \min (1+2 \alpha / n, 2)$. In particular, we may always take $w \in A_{1}$, and if $n=1$ and $\frac{1}{2} \leq \alpha<1$, we may take $w \in A_{2}$.

Proof of Lemma 2.1. This is fairly trivial. First (see, e.g., Stein [S, pp. 162-163, $6.12,6.13$ ] and the references given therein) we have the pointwise bound

$$
g_{\alpha} f(x) \leq C_{\alpha, \lambda} g_{\lambda}^{*} f(x)
$$

if $\lambda<1+2 \alpha / n$ (see [S, p. 88] for the definition of $g_{\lambda}^{*}$ ). But by a result of Muckenhoupt and Wheeden [MW], $g_{\lambda}^{*}$ is bounded on $L_{w}^{2}$, with $w \in A_{q(\lambda)}$, $q(\lambda) \equiv \min (\lambda, 2), \lambda>1$. If $1+2 \alpha / n>2$, we take $\lambda=2$. If $1+2 \alpha / n \leq 2$ and $w \in A_{1+2 \alpha / n}$, then by a well-known property of $A_{p}$ weights we may select a $\lambda<1+2 \alpha / n$, with $w \in A_{\lambda}$. In either case, Lemma 2.1 follows by [MW] and the pointwise bound (2.2).

We now give a weighted version of Theorem (3.3) of [Stz].

Lemma 2.3. Suppose $0<\alpha<1$ and $A=I_{\alpha} a, a \in B M O$. If $Q(s)$ is a cube with side length $s$ and $p(\alpha) \equiv \min (1+2 \alpha / n, 2)$, then

$$
\frac{1}{w(Q(s))} \int_{Q(s)} \int_{|h| \leq s} \frac{|A(x+h)-A(x)|^{2}}{|h|^{n+2 \alpha}} d h w(x) d x \leq C_{\alpha}\|a\|_{*}^{2},
$$

where $w \in A_{p(\alpha)}$. In particular, we may always take $w \in A_{1}$, and if $\frac{1}{2} \leq \alpha<1$ and $n=1$, we may take $w \in A_{2}$.

Proof of Lemma 2.3. This is easy if we follow the argument in [Stz], combined with that of Journe [J, pp. 85-87], so we only give a brief sketch. Since the operator

$$
f \rightarrow I_{\alpha} f(x+h)-I_{\alpha} f(x)
$$

has Fourier multiplier $\left[e^{i h \cdot \xi}-1\right]|\xi|^{-\alpha}$, it annihilates constants, so we may assume that $a$ has mean value zero on $Q^{*}(s)$. Here $Q^{*}(s)$ denotes the cube concentric with $Q(s)$ and has ten times the diameter of $Q(s)$. As usual, we write $a=a_{1}+a_{2}$, where $a_{1} \equiv a \chi_{Q^{*}(s)}, a_{2}=a \chi_{\left(Q^{*}(s)\right)} c$. Now crudely, by Lemma 2.1, the left side of (2.4) with $I_{\alpha} a_{1}$ in place of $A$ is no larger than a constant times

$$
\frac{1}{w(Q(s))} \int\left|a_{1}(x)\right|^{2} w(x) d x
$$

The desired estimate for this last term may be obtained exactly like the corresponding estimate in [J, p. 86] by using Hölder's inequality, the reverse Hölder property of $A_{p}$ weights, the John-Nirenberg Theorem, and the fact that $w$ defines a doubling measure. To handle the part of (2.4) corresponding to $I_{\alpha} a_{2}$, 
we observe first that the operator defined by $(2.5)$ is given by convolution with the kernel

$$
C_{n}\left[\frac{1}{|x+h|^{n-\alpha}}-\frac{1}{|x|^{n-\alpha}}\right] \leq C_{n, \alpha} \frac{|h|}{|x|^{n+1-\alpha}},
$$

where the last inequality holds whenever $|x|>2|h|$. If we write $h=t \theta$ in polar coordinates, then the left side of (2.4) with $I_{\alpha} a_{2}$ in place of $A$ is bounded by

$$
\int_{S^{n-1}} \int_{Q(s)} \int_{0}^{s}\left[\int_{\mathbb{R}^{n}} \frac{t^{1-\alpha}}{(t+|x-y|)^{n+1-\alpha}}\left|a_{2}(y)\right| d y\right]^{2} \frac{d t}{t} w(x) d x d \theta .
$$

For $x \in Q(s)$ and $y \in\left(Q^{*}(s)^{c}\right.$, we have $(s+|x-y|) \approx|x-y| \approx(t+|x-y|)$. Thus, the expression in square brackets in $(2.6)$ is dominated by a constant times

$$
\left(\frac{t}{s}\right)^{1-\alpha} \int_{\mathbb{R}^{n}} \frac{s^{1-\alpha}}{(s+|x-y|)^{n+1-\alpha}}\left|a_{2}(y)\right| d y \leq C\left(\frac{t}{s}\right)^{1-\alpha}\|a\|_{*},
$$

where the last inequality follows by a slight variant of a classical argument of Fefferman Stein [FS] (see, e.g., [Stz, Lemma 2.2]). Lemma 2.3 may now be obtained by plugging this last expression into (2.6).

\section{Proof of Theorem 1.4}

The proof is based on ideas developed by Lewis and Murray in [LM, §3]. Our objective is to prove

$$
\int_{\mathbb{R}} \int_{0}^{\infty}\left|Q_{s} K_{\alpha} f(x)\right|^{2} \frac{d s}{s^{1+2 \alpha}} w(x) d x \leq C_{\alpha} \int_{\mathbb{R}}|f(x)|^{2} w(x) d x,
$$

where, without loss of generality, we assumed that $\|a\|_{*}=1$ (recall that $A=$ $\left.I_{\alpha} a, a \in \mathrm{BMO}\right)$. Here, $w \in A_{2}$ if $\frac{1}{2} \leq \alpha<1$, or $w \in A_{1}$ if $0<\alpha<1$. We smoothly truncate the kernel of $K_{\alpha}$ as follows. Choose a radial $\varphi \in C_{0}^{\infty}$, $0 \leq \varphi \leq 1$, where $\varphi \equiv 1$ on $\{|x|<100\}$ and $\varphi \equiv 0$ on $\{|x|>101\}$. For fixed $s$, we write

$$
\begin{aligned}
\frac{[A(x)-A(y)]^{2}}{|x-y|^{1+\alpha}} \equiv[A(x)-A(y)]^{2}\left\{|x-y|^{-1-\alpha} \varphi\left(\frac{|x-y|}{s}\right)\right. \\
\left.\quad+|x-y|^{-1-\alpha}\left(1-\varphi\left(\frac{|x-y|}{s}\right)\right)\right\} \\
\equiv[A(x)-A(y)]^{2}\left\{j_{s}(x-y)+k_{s}(x-y)\right\} .
\end{aligned}
$$

We consider first the term corresponding to $j_{s}$, which is essentially the same as $\theta_{1}$ in [LM, (3.10)] (the term $\theta_{2}$ in [LM] will not arise in the present argument and their term $\theta_{3}$ corresponds to $k_{s}$ ). The part of the left side of $(3.1)$ corresponding to $j_{s}$ is crudely bounded by

$$
\int_{0}^{\infty} \int_{\mathbb{R}}\left[\int_{\mathbb{R}} \int_{\mathbb{R}}\left|\psi_{s}(x-z)\right|(A(z)-A(y))^{2} j_{s}(z-y)|f(y)| d z d y\right]^{2} w(x) d x \frac{d s}{s^{1+2 \alpha}} .
$$

Since convolution with $\left|\psi_{s}\right|$ is controlled by the Maximal Function, by Muckenhoupt's theorem the last expression is no larger than a constant times

$$
\int_{0}^{\infty} \int_{\mathbb{R}}\left[\int_{\mathbb{R}}(A(x)-A(y))^{2} j_{s}(x-y)|f(y)| d y\right]^{2} w(x) d x \frac{d s}{s^{1+2 \alpha}}
$$


for all $w \in A_{2}$. In analogy with [LM, (3.15)-(3.17)], we apply Minkowski's integral inequality to obtain the bound

$$
\begin{aligned}
& \int_{\mathbb{R}}\left(\int \frac{A(x)-A(y)^{2}}{|x-y|^{1+\alpha}}|f(y)|\left(\int_{|x-y| / 101}^{\infty} \frac{d s}{s^{1+2 \alpha}}\right)^{1 / 2} d y\right)^{2} w(x) d x \\
& \quad=C_{\alpha}\left\|C_{2}(|f|)\right\|_{2, w}^{2},
\end{aligned}
$$

where $C_{2}$ is the second-order fractional commutator with kernel

$$
k^{(2)}(x, y) \equiv \frac{(A(x)-A(y))^{2}}{|x-y|^{1+2 \alpha}} .
$$

But $C_{2}$ is bounded on unweighted $L^{2}$ by a result of Murray [M2]. Furthermore, since $A \in I_{\alpha}(\mathrm{BMO}) \subseteq \operatorname{Lip} \alpha$, the kernel $k^{(2)}$ satisfies "standard" CalderonZygmund estimates, so $C_{2}$ is bounded on $L_{w}^{2}, w \in A_{2}$, by the usual arguments (see, e.g., Coifman and Fefferman [CF]).

We now turn to the part of (3.1) corresponding to $k_{s}$. We consider the kernel of the composition of $Q_{s}$ with the operator

$$
f \rightarrow \int[A(x)-A(y)]^{2} k_{s}(x-y) f(y) d y .
$$

Since $Q_{s} 1=0$, this kernel equals

$$
\begin{aligned}
\int_{\mathbb{R}} \psi_{s}( & x-z)[A(z)-A(y)]^{2}\left[k_{s}(z-y)-k_{s}(x-y)\right] d z \\
& +\int_{\mathbb{R}} \psi_{s}(x-z)\left([A(z)-A(y)]^{2}-[A(x)-A(y)]^{2}\right) k_{s}(x-y) d z \\
\equiv & H_{s}(x, y)+L_{s}(x, y) .
\end{aligned}
$$

The terms $H_{s}$ and $L_{s}$ correspond to $\sigma_{1}$ and $\sigma_{2}$ in [LM, (3.24) and (3.25)]. We treat $L_{s}$ first, and following [LM, (3.32)] we write

$$
\begin{aligned}
& (A(z)-A(y))^{2}-(A(x)-A(y))^{2} \\
& \quad=(A(z)-A(x))^{2}+2(A(z)-A(x))(A(x)-A(y)) .
\end{aligned}
$$

Since $\int \psi=0$, the part of $L_{s}$ containing the second part of (3.5) equals twice

$$
Q_{s} A(x)[A(x)-A(y)] k_{s}(x-y) .
$$

Recall that $A=I_{\alpha} a$, with $a \in \mathrm{BMO}$. Plugging (3.6) into (3.1) in place of $Q_{s} K_{\alpha}$, we obtain

$$
\int_{\mathbb{R}} \int_{0}^{\infty}\left|\widetilde{Q}_{s} a(x) C_{1,100 s} f(x)\right|^{2} \frac{d s}{s} w(x) d x,
$$

where $\widetilde{Q}_{s} \equiv s^{-\alpha} Q_{s} I_{\alpha}$ and $C_{1,100 s}$ is the smoothly truncated first fractional commutator with kernel

$$
\frac{A(x)-A(y)}{|x-y|^{1+\alpha}}\left(1-\varphi\left(\frac{|x-y|}{s}\right)\right) .
$$

But $\left|\widetilde{Q}_{s} a(x)\right|^{2} w(x) \frac{d s}{s} d x$ is a weighted Carleson measure for all $w \in A_{2}$ (see [J, pp. 85-87]), so by a standard argument (3.7) is no larger than $\left\|N\left(C_{1,100 s} f\right)\right\|_{2, w}^{2}$, where $N$ is the nontangential maximal operator $N g_{s}\left(x_{0}\right) \equiv$ 
$\sup _{\left|x-x_{0}\right|<s}\left|g_{s}(x)\right|$. Now $C_{1}$ is bounded on $L^{2}$ by [M1], and since the kernel $(A(x)-A(y))|x-y|^{-1-\alpha}$ satisfies "standard" Calderon-Zygmund estimates, the corresponding maximal singular integral

$$
C_{1, *} f \equiv \sup _{s>0}\left|C_{1,100 s} f\right|
$$

is bounded on $L_{w}^{2}, w \in A_{2}$. Thus, as in [LM, (3.39)], the nontangential maximal function $N\left(C_{1,100 s} f\right)$ is bounded on $L_{w}^{2}$. In fact, the observation in [LM] holds for any Calderon-Zygmund operator $T$ with "standard" kernel $k(x, y)$, since for $\left|x-x_{0}\right|<s$, we have

$$
\begin{aligned}
\left|T_{100 s} f(x)\right| \leq & \int\left|k(x, y) \Phi(|x-y| / s)-k\left(x_{0}, y\right) \Phi\left(\left|x_{0}-y\right| / s\right)\right||f(y)| d y \\
& +T_{*} f\left(x_{0}\right),
\end{aligned}
$$

where $\Phi=1-\varphi$. The first term on the right side of (3.8) is no larger than

$$
C \int \frac{s^{\varepsilon}}{\left(s+\left|x_{0}-y\right|\right)^{n+\varepsilon}}|f(y)| d y \leq C M f\left(x_{0}\right),
$$

by the standard kernel conditions for $k(x, y)$.

Next, we consider the part of $L_{s}$ containing the first term in (3.5). We need to estimate

$$
\int_{\mathbb{R}} \int_{0}^{\infty}\left|\int_{\mathbb{R}} \psi_{s}(x-z)[A(z)-A(x)]^{2} d z \int_{\mathbb{R}} k_{s}(x-y) f(y) d y\right|^{2} \frac{d s}{s^{1+2 \alpha}} w(x) d x .
$$

Now, $\left|k_{s}(x-y)\right| \leq C /(|h|+|x-y|)^{1+\alpha}$ for $|h|<s$. Furthermore, $A \in \operatorname{Lip}_{\alpha}$, so by the change of variables $z \rightarrow z+x$, and then $z \rightarrow s z$, we have that (3.9) is bounded by

$$
\int_{\mathbb{R}} \int_{0}^{\infty}\left(\int_{|z|<1}|A(x+s z)-A(x)| P_{s|z|}(|f|)(x) d z\right)^{2} \frac{d s}{s^{1+2 \alpha}} w(x) d x,
$$

where $P_{t}$ denotes convolution with the kernel $t^{\alpha} /(t+|x|)^{1+\alpha}$. Now by Minkowski's integral inequality, the square root of $(3.10)$ is no larger than

$$
\int_{|z|<1}\left(\int_{\mathbb{R}} \int_{0}^{\infty}|A(x+s z)-A(x)|^{2}\left(P_{s|z|}(|f|)(x)\right)^{2} \frac{d s}{s^{1+2 \alpha}} w(x) d x\right)^{1 / 2} d z
$$

The desired estimate now follows by the change of variable $s \rightarrow s /|z|$, and a standard argument using the weighted Carleson measure condition (2.4), and the fact that the nontangential maximal function $N\left(P_{s} f\right)$ is bounded on $L_{w}^{2}$, $w \in A_{2}$.

To conclude the proof of Theorem 1.4, it remains to consider $H_{s}$ in (3.4). The part of (3.1) corresponding to this term is dominated by

$$
\begin{aligned}
\int_{0}^{\infty} \int_{\mathbb{R}}\left(\int_{\mathbb{R}} \int_{\mathbb{R}}\left|\psi_{s}(x-z)\right|(A(z)-A(y))^{2} \frac{s}{|z-y|^{2+\alpha}}\right. \\
\times \chi\{|z-y|>99 s\}|f(y)| d y d z)^{2} w(x) d x \frac{d s}{s^{1+2 \alpha}},
\end{aligned}
$$


where we have applied the mean value theorem to $k_{s}$ and used the fact that $|x-z|<s$. For $w \in A_{2}$, again by Muckenhoupt's Theorem, this last expression is no larger than a constant times

$$
\begin{aligned}
\int_{0}^{\infty} \int_{\mathbb{R}}\left(\int_{\mathbb{R}} \frac{(A(x)-A(y))^{2}}{|x-y|^{1+2 \alpha}}\left(\frac{s}{|x-y|}\right)^{1-\alpha}\right. \\
\times \chi\{|x-y|>99 s\}|f(y)| d y)^{2} w(x) d x \frac{d s}{s} .
\end{aligned}
$$

By Minkowski's integral inequality, we have the bound

$$
\begin{aligned}
& \int_{\mathbb{R}}\left(\int_{\mathbb{R}} \frac{A(x)-A(y))^{2}}{|x-y|^{1+2 \alpha}}|f(y)|\left(\int_{0}^{|x-y| / 99}\left(\frac{s}{|x-y|}\right)^{2(1-\alpha)} \frac{d s}{s}\right)^{1 / 2} d y\right)^{2} w(x) d x \\
& \quad \leq C\left\|C_{2}(|f|)\right\|_{2, w}^{2},
\end{aligned}
$$

and the theorem follows.

\section{EXTENSION TO THE SINGLE LAYER POTENTIAL}

Consider first the modified single layer potential

$$
S_{\alpha} f(x) \equiv \int_{\mathbb{R}} W_{\alpha}(x, y) f(y) d y,
$$

where

$$
W_{\alpha}(x, y) \equiv|x-y|^{\alpha-1} \exp \left[-\frac{\left(A(x)-A(y)^{2}\right.}{|x-y|^{2 \alpha}}\right] .
$$

We have the following weighted version of [LM, Theorem 2] (see also [LM, Theorems 4 and 5]).

Theorem 4.1. Let $A=I_{\alpha} a$, and suppose $w \in A_{2}$ if $\frac{1}{2} \leq \alpha<1$, or $w \in A_{1}$ if $0<\alpha<1$. Then

$$
\left\|\left(S_{\alpha}-c_{\alpha} I_{\alpha}\right) f\right\|_{I_{\alpha}\left(L_{w}^{2}\right)} \leq C_{\alpha}\left(\|a\|_{*}^{2}+\|a\|_{*}^{4}\right)\|f\|_{2, w} .
$$

Proof of Theorem 4.1. We will follow [LM, Theorem 2] and obtain the theorem by an easy modification of the proof of Theorem 1.4. The operator $S_{\alpha}-c_{\alpha x} I_{\alpha}$ has kernel

$$
|x-y|^{\alpha-1}\left\{\exp \left[-\frac{(A(x)-A(y))^{2}}{|x-y|^{2 \alpha}}\right]-1\right\} .
$$

The expression in curly brackets in (4.2) is no larger than a constant times $[A(x)-A(y)]^{2}|x-y|^{-2 \alpha}$, so if we multiply (4.2) by a smooth radial cut-off factor $\varphi(|x-y| / s)$, then we get a term that can be handled exactly like the term corresponding to $j_{s}$ in (3.2). It therefore remains to treat (4.2) times

$$
\Phi\left(\frac{|x-y|}{s}\right) \equiv\left(1-\varphi\left(\frac{|x-y|}{s}\right)\right) .
$$

As in (3.4), we must consider the following analogues of (3.47) and (3.48) in [LM]:

$$
\begin{aligned}
& \int_{\mathbb{R}} \psi_{s}(x-z)\left\{\exp \left[-\frac{(A(z)-A(y))^{2}}{|z-y|^{2 \alpha}}\right]-1\right\} \\
& \quad \times\left[|z-y|^{\alpha-1} \Phi\left(\frac{|z-y|}{s}\right)-|x-y|^{\alpha-1} \Phi\left(\frac{|x-y|}{s}\right)\right] d z,
\end{aligned}
$$




$$
\begin{aligned}
\int_{\mathbb{R}} \psi_{s}(x-z)\left\{\exp \left[-\frac{(A(z)-A(y))^{2}}{|z-y|^{2 \alpha}}\right]\right. & \left.-\exp \left[-\frac{(A(x)-A(y))^{2}}{|x-y|^{2 \alpha}}\right]\right\} \\
& \times|x-y|^{\alpha-1} \Phi\left(\frac{|x-y|}{s}\right) d z .
\end{aligned}
$$

These correspond to $H_{s}$ and $L_{s}$ in (3.4) respectively. The former can be handled exactly as before; in fact, we obtain the same upper bound (3.11).

Next, by Taylor's theorem the expression in curly brackets in (4.4) equals

$$
\begin{gathered}
-\left[\frac{A(z)-A(y))^{2}}{|z-y|^{2 \alpha}}-\frac{(A(x)-A(y))^{2}}{|x-y|^{2 \alpha}}\right] \exp \left[-\frac{(A(x)-A(y))^{2}}{|x-y|^{2 \alpha}}\right], \\
+\left[\frac{(A(z)-A(y))^{2}}{|z-y|^{2 \alpha}}-\frac{(A(x)-A(y))^{2}}{|x-y|^{2 \alpha}}\right]^{2} E(x, y, z),
\end{gathered}
$$

where $0 \leq E(x, y, z) \leq 1$. By analogy to (3.4) and (3.5),

$$
\begin{aligned}
\frac{(A(z)-A(y))^{2}}{|z-y|^{2 \alpha}}-\frac{(A(x)-A(y))^{2}}{|x-y|^{2 \alpha}} & \\
= & (A(z)-A(y))^{2}\left[\frac{1}{|z-y|^{2 \alpha}}-\frac{1}{|x-y|^{2 \alpha}}\right] \\
& \quad+\frac{(A(z)-A(x))^{2}}{|x-y|^{2 \alpha}}+\frac{2(A(z)-A(x))(A(x)-A(y))}{|x-y|^{2 \alpha}} \\
\equiv & B_{1}(x, y, z)+B_{2}(x, y, z)+B_{3}(x, y, z) .
\end{aligned}
$$

Since $|x-z|<s \ll|x-y|$ (so, in particular, $|x-y| \approx|z-y|$ ), we can handle the part of (4.5) corresponding to $B_{1}$ exactly like $H_{s}$ in (3.4) (see (3.11)). Since (4.6) is no larger than $C E(x, y, z) \sum_{i=1}^{3}\left(B_{i}(x, y, z)\right)^{2}$, and since trivially

$$
\left|B_{1}\right| \leq C\|A\|_{\text {Lip } \alpha}^{2} \leq C\|a\|_{*}^{2},
$$

the same reasoning applies to the parts of (4.6) corresponding to $B_{1}$. Similarly, those parts of (4.5) and (4.6) involving $B_{2}$ may be treated exactly like the first term in (3.5) (see (3.9), (3.10), and the related discussion). The latter argument also applies to the term $\left(B_{3}(x, y, z)\right)^{2} E(x, y, z)$ arising in (4.6).

Thus, it remains only to consider the following part of (4.5): $B_{3}(x, y, z)$ $\times \exp \left[-(A(x)-A(y))^{2} /|x-y|^{2 \alpha}\right]$ (we have ignored multiplication by -2 ). This is the only term where we do not reduce matters to the treatment of an appropriate positive operator, so the presence of a bounded, nonconstant multiplicative factor can no longer be ignored. If we plug this last expression into (4.4) in place of \{\} and let the corresponding operator act on a function $f$, then we get (since $\int \psi=0$ )

$$
Q_{s} A(x) T_{100 s} f(x) \text {, }
$$

where $T_{100 s}$ has the (truncated) standard kernel

$$
\frac{(A(x)-A(y))}{|x-y|^{1+\alpha}} \exp \left[-\frac{(A(x)-A(y))^{2}}{|x-y|^{2 \alpha}}\right] \Phi\left(\frac{|x-y|}{s}\right) \text {. }
$$

As before (see (3.7), (3.8), and the related discussion), the theorem will follow by weighted Carleson measure theory once we show that the maximal singular integral

$$
T_{*} f \equiv \sup _{s>0}\left|T_{100 s} f\right|
$$


is bounded on $L_{w}^{2}, w \in A_{2}$. But this is easy since the mean value theorem gives

$$
\exp \left[-\frac{(A(x)-A(y))^{2}}{|x-y|^{2 \alpha}}\right]=1+\left[\frac{(A(x)-A(y))^{2}}{|x-y|^{2 \alpha}}\right] \widetilde{E}(x, y),
$$

with $|\widetilde{E}| \leq 1$. The term corresponding to 1 is just the first fractional commutator $C_{1}$, and the term corresponding to the second part of the right side of (4.8) is no larger than $C\|A\|_{\operatorname{Lip} \alpha} C_{2}(|f|)$, and we are done.

In conclusion, we remark that as in [LM], a straightforward modification of the above arguments enables one to multiply the kernels that we have considered (e.g., (4.2) or (1.3)) by $\chi\{x-y>0\}$. In particular, for $\alpha=\frac{1}{2}$, we can treat the boundary single layer potential for the heat equation for all $w \in A_{2}$ and thus for all $p, 1<p<\infty$.

\section{ACKNOWLEDGMENTS}

I am very grateful to Margaret A. M. Murray for kindly providing me with a preprint of her joint work with J. L. Lewis. I also thank Russell Brown, who proposed the problem of finding a simpler proof of the results of [LM].

\section{BIBLIOGRAPHY}

[CF] R. Coifman and C. Fefferman, Weighted norm inequalities for maximal functions and singular integrals, Studia Math. 51 (1974), 241-250.

[CR] R. Coifman and R. Rochberg, Another characterization of BMO, Proc. Amer. Math. Soc. 79 (1980), 249-254.

[FS] C. Fefferman and E. M. Stein, $H^{p}$ spaces of several variables, Acta Math. 129 (1971), 137-193.

[GR] J. Carcia-Cuerva and J. L. Rubio de Francia, Weighted inequalities and related topics, NorthHolland, Amsterdam, 1985.

[J] J. L. Journe, Calderon-Zygmund operators, pseudo-differential operators, and the Cauchy integral of Calderon, Lecture Notes in Math., vol. 994, Springer-Verlag, Berlin, 1983.

[LM] J. L. Lewis and M. A. M. Murray, Regularity properties of commutators and layer potentials associated to the heat equation, Trans. Amer. Math. Soc. 328 (1991), 815-842.

[MW] B. Muckenhoupt and R. Wheeden, Norm inequalities for the Littlewood-Paley $g_{\lambda}^{*}$ function, Trans. Amer. Math. Soc. 191 (1974), 95-111.

[M1] M. A. M. Murray, Commutators with fractional differentiation and BMO Sobolev spaces, Indiana Univ. Math. J. 34 (1985), 205-215.

[M2] _ Multilinear singular integrals involving a derivative of fractional order, Studia Math. 87 (1987), 139-165.

[S] E. M. Stein, Singular integrals and differentiability properties of functions, Princeton Univ. Press, Princeton, NJ, 1970.

[Stz] R. Strichartz, Bounded mean oscillation and Sobolev spaces, Indiana Univ. Math. J. 29 (1980), 539-558.

Department of Mathematics and Statistics, Wright State University, Dayton, Ohio 45435 